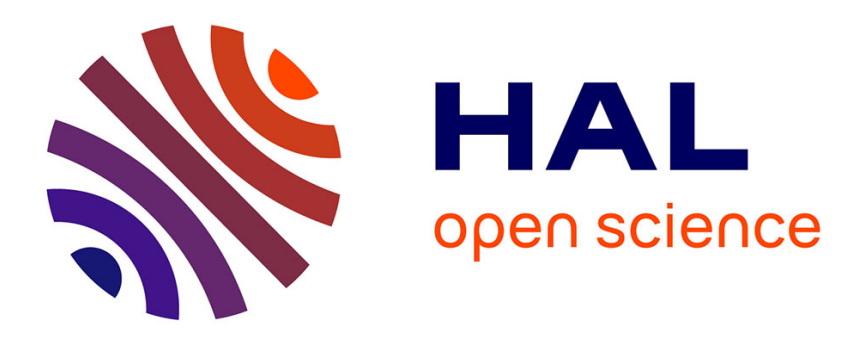

\title{
Ouvrir l'Université aux possibles démocratiques
}

Fabrice Flipo, Lionel Larqué

\section{To cite this version:}

Fabrice Flipo, Lionel Larqué. Ouvrir l'Université aux possibles démocratiques. Revue du MAUSS, 2009, 33, pp.306-326. 10.3917/rdm.033.0306 . hal-00957952

\section{HAL Id: hal-00957952 https://hal.science/hal-00957952}

Submitted on 14 Mar 2014

HAL is a multi-disciplinary open access archive for the deposit and dissemination of scientific research documents, whether they are published or not. The documents may come from teaching and research institutions in France or abroad, or from public or private research centers.
L'archive ouverte pluridisciplinaire HAL, est destinée au dépôt et à la diffusion de documents scientifiques de niveau recherche, publiés ou non, émanant des établissements d'enseignement et de recherche français ou étrangers, des laboratoires publics ou privés. 


\title{
Ouvrir l'Université aux possibles démocratiques
}

\author{
Fabrice Flipo*, Lionel Larqué** \\ * philosophe, maître de conférences à TELECOM \& Management SudParis fabrice.flipo@it- \\ sudparis.eu \\ ** association Les Petits Débrouillards
}
Fabrice Flipo et Lionel Larqué sont administrateurs de la Fondation Sciences Citoyennes www.sciencescitoyennes.org

\section{Remerciements : Françoise Gollain}

La question de l'université est souvent envisagée au travers de ses relations avec le pouvoir ou de ses liens avec la société en tant que destinataire de savoirs élaborés par des spécialistes. Cette position dominante est aujourd'hui mise en question par la société. Le pouvoir utilise cet écart croissant entre base et université en tant que corps intermédiaire pour essayer de la mettre au pas. L'indépendance du savoir et de la pensée est essentielle dans une démocratie. Le risque de lyssenkisme ou d'anti-intellectualisme est réel, mais asseoir l'indépendance et l'autonomie sur une gestion en cercle fermé (" entre pairs ») accroît l'écart avec la société. Pour préserver l'autonomie, l'université doit au contraire chercher des appuis dans la société en tant que corps politique. Cet article tente d'élaborer les termes d'une telle démocratisation.

The question of the university is often envisaged through its relations with the power or through its links with the society as addressee of knowledges elaborated by specialists. This dominant position is questioned by the society today. The power uses this distance growing between base and university as intermediate body to try to bring it in to heel. The independence of the knowledge and the thought is essential in a democracy. The risk of lyssenkisme or anti-intellectualism is real, but to base the independence and the autonomy on a management in closed circle (« between peers ») increases the distance with the society. On the contrary, to ensure a real autonomy, the university has to look for support in the society as political body. This article tries to elaborate the terms of such a democratization.

\section{La question des finalités de l'université}

Nicolas Sarkozy s'en est pris à l'université sur la base de deux critiques : un supposé manque de travail de la part des enseignants-chercheurs et une qualité insuffisante, ceci se traduisant principalement par un manque de compétitivité internationale - la contribution à la " société de la connaissance ». La méthode est désormais classique : jouer la France d'en bas, celle qui travaille contre les corps intermédiaires, supposés profiter de situations de rente; mettre aux commandes la France d'en haut (Présidents etc.), jugée dépositaire de qualités exceptionnelles et preuve vivante de la méritocratie - sans, bien sûr, que l'évaluation de ces personnalités ne se pose jamais vraiment.

Le mouvement des enseignants-chercheurs $(\mathrm{EC})$ a répondu de manière convaincante en arguant pour l'essentiel de trois arguments ${ }^{1}$. Le manque de moyens, tout d'abord, vient au premier rang des revendications : la méritocratie est en panne car les moyens sont inégalement ou mal distribués. Une augmentation significative du taux d'encadrement permettrait d'apporter un des éléments de réponse les plus efficaces à ce défi. Dans le domaine de la recherche et des programmes, ensuite, le souci de maintenir "l'indépendance ». La justification ici est que

1 Voir la vidéo qui circule quant au nombre de publis, brevets etc. par exemple ici

http://www.agoravox.tv/article.php3?id_article=21792 
l'université doit produire et diffuser le savoir et non se transformer en agence de formation continue visant avant tout l'insertion professionnelle, ni en "idéologie » susceptible de provoquer des dérives lyssenkistes. Enfin les chercheurs ont défendu leurs résultats et démontré la bonne tenue de la France sur le plan de la compétitivité et des publications ${ }^{2}$ - malgré le manque de moyens.

En agissant ainsi, le mouvement des EC, via les syndicats et de nouveaux acteurs dits hybrides comme SLU, a inscrit la crise actuelle dans la forme de revendications catégorielles. Cela lui a certes permis d'atteindre une certaine lisibilité ${ }^{3}$, et d'obtenir plusieurs succès : Valérie Pécresse a déjà perdu dans les faits. La majorité des organisations estime que cette ligne de défense suffit ${ }^{4}$. C'est oublier un enjeu qui avait bien été pointé lors des Etats Généraux de la recherche à Grenoble il y a un peu plus de 4 ans $^{5}$ : l'orientation de la recherche et de l'enseignement, autrement dit la question de leur contenu. Ici la ligne de défense du mouvement des EC tient à un seul mot: "l'indépendance » (ou " autonomie »). Mais comment les EC entendent-ils garantir cette " indépendance » ? Indépendance par rapport à quoi ? Pour l'essentiel, cette indépendance se définit par un double souci : ne dépendre ni de l'Etat (pouvoir politique), d'une part, car l'Etat est toujours suspecté de vouloir bâillonner les EC ou chercher à provoquer des dérives lyssenkistes, ni des financements issus du privé, d'autre part, ces derniers étant perçus comme étant trop " appliqués » et peu soucieux des enjeux de long terme. Ce qui nous fait donc deux critères : la science doit être produite et enseignée sur deniers publics, d'une part, et la science contrôler la science, d'autre part.

Le problème est que ces deux arguments risquent de ne pas être suffisants. Ils permettent certes de relier le combat de la recherche avec celui des services publics, mais ils prêtent le flanc aux mêmes critiques, des critiques qui ont jusqu'ici eu assez de poids pour faire passer les réformes précédentes dans l'opinion puis dans les faits, que ces réformes aient été initiées par la gauche ou par la droite : privatisation de la Poste, libéralisation de l'électricité etc.

\section{Le savoir en action}

Quel résultat veut-on atteindre, finalement? Peut-on démontrer que les financements publics et la gouvernance " entre pairs » est la meilleure garantie de la liberté de penser, d'expérimenter, de chercher, indépendante de tous les pouvoirs ? Examinons la situation à l'aune des 5 tâches définissant classiquement le métier d'EC : élaborer des connaissances "scientifiques ", diffuser, former, valoriser, expertiser ${ }^{6}$. Cette définition de base va nous permettre de faire quelques distinctions utiles pour la suite.

Remarquons tout d'abord que l'université n'a pas le monopole de ces cinq tâches : l'ensemble du corps social s'y adonne - à commencer par les parents envers leurs enfants comme les acteurs de l'éducation non formelle. Quelle est alors la spécificité de l'EC ? Est-ce de former, diffuser et transmettre des savoirs "plus vrais » que les savoirs populaires ? Mais qu'est-ce que cette "vérité "? La sociologie des sciences a montré non seulement l'important décalage qui peut exister entre les professionnels spécialisés et les citoyens, experts de leur quotidien - mais aussi que la plus grande adéquation aux faits n'étant pas toujours du côté des professionnels ${ }^{7}$. Le savoir des EC est organisé en disciplines, dont l'émiettement pose des problèmes qui ont maintes fois été soulignés - et qui sont encore parfaitement d'actualité. Le régime de la preuve est bien souvent endogène à une discipline donnée, qui oublie ses axiomes fondateurs (Lakatos). La lutte des scientifiques pour l'hégémonie intellectuelle, le fait que les scientifiques sont sujets aux paradigmes - cf. les travaux de Thomas Kuhn - y compris dans le domaine de la charge de la preuve. Les sciences s'accusent entre elles de manque de scientificité : les chercheurs en sciences dures se moquent facilement de la " mollesse " des "preuves " des sciences humaines

2 Voir les revendications des syndicats, de SLR etc.

3 Voir D. Cefaï, Pourquoi se mobilise-t-on ?, Paris, La Découverte, 2007 sur les revendications collectives et l'économie de mise en forme faite par les mouvements quand ils réactivent des schémas de mobilisation connus

4 Voir les revendications SLR, syndicats etc.

5 Rapport des Etats Généraux de la Recherche, novembre 2004. http://cip-etats-generaux.apinc.org/

6 EGR 2004

7 M. Callon, P. Lascoumes \& Y. Barthe, Agir dans un monde incertain, Paris, Seuil, 2003. 
tandis que ces dernières se gaussent facilement de la naïveté des sciences dures quant à leurs propres fondements - des querelles ont récemment été ravivées par la sociologie des sciences mais aussi l'histoire des sciences, justement ${ }^{8}$. Dans tous les cas, les savoirs spécialisés, pour avoir une quelconque vertu critique, sont soumis à un délicat problème de "traduction » vers le " monde de la vie " au sens de Husserl ${ }^{9}$. Ce que révèlent aussi les controverses autour des OGM, nanotechnologies, nucléaire etc. est à quel point les EC séparent mal la "science " de "l'expertise ». La science est une connaissance qui a elle seule ne permet pas de tirer de conclusions pour l'action, cette dernière étant d'ordre moral et politique. Pourtant les EC, même dépendants de fonds publics, s'autorisent régulièrement à tirer des conclusions pour l'action court-circuitant ainsi le débat proprement politique. Ils franchissent parfois allègrement la frontière ténue qui peut exister entre science et science-fiction, pour donner dans le " hype » et les promesses prométhéennes ${ }^{10}$. En cela leur incompréhension entre les rôles de scientifiques et d'experts est manifeste ${ }^{11}$. Les associations de la société civile s'opposent régulièrement aux EC dont l'expertise est "formelle ${ }^{12}$ et ne souffre aucun débat. Le débat ne descend pas de ce niveau, le peuple est pris de haut, il est théorisé comme une partie " profane » - les EC se considérant donc implicitement comme relevant du "sacré »- alors que les enjeux sont souvent de l'ordre de choix de société, aux conséquences majeures. De quelle " indépendance » s'agit-il, alors ? Le statut, le salaire et la critique par les pairs ne semblent pas en mesure de garantir les errances des EC et de leur communauté spécialisée.

Un autre point mérite d'être souligné. Le savoir est immatériel, l'économie du même nom l'a assez souligné. Mais contrairement à ce que "l'économie de la connaissance " voudrait nous faire croire, en ne s'intéressant qu'à un type de savoir très particulier, celui qui est "économiquement productif », la duplication du savoir est rarement à coût nul, et sa transmission n'enrichit que si elle ne conduit pas à détruire d'autres savoirs. Les savoirs sont toujours des savoir-faire ${ }^{13}$, penser le monde c'est le découper et l'agencer. La transmission et plus généralement la mémorisation des savoirs impliquent l'existence et l'entretien de supports matériels ${ }^{14}$ dont le poids économique et budgétaire s'il est loin d'être négligeable tend même à s'alourdir notablement ${ }^{15}$. Les moyens n'étant pas infinis, ce qui sera investi ici (en physique des particules, mathématiques, sociologie etc.) ne le sera pas là (physique du climat, philosophie etc.). Les milliards dépensés dans ITER ou le LHC (Large Hadron Collider, accélérateur de particules qui a coûté près de 2 milliards d'euros) sont des moyens qui auraient pu être affectés à d'autres usages. Les arbitrages sont parfois douloureux et toujours avec des conséquences sur la production de connaissances Toutes les disciplines et tous les savoirs sont intéressants, dans le ciel des idées immatérielles, mais dans le concret les sociétés font des choix, elles conservent, innovent et oublient. Et dans ce domaine on voit mal quelles garanties la discussion au sein d'un nombre réduit de pairs peut offrir. De tels enjeux semblent plutôt devoir être décidés par la société entière.

Un point mérite d'être éclairci à ce stade. Nous ne défendons ici aucune conception " relativiste » du savoir et de la vérité, au sens d'un constructivisme qui serait absolu. II existe bel et bien des faits qui permettent à quelque chose comme une objectivité d'avoir lieu. Mais les savoirs ont des conditions de validité déterminées, et ces conditions ne sont pas indépendantes du temps ni de l'espace. Dans l'ordre du temps, les savoirs sont dépendants de l'innovation et de l'oubli, des paradigmes etc. Dans l'ordre de l'espace, les savoirs dépendent pour partie des lieux (tel écosystème ou telle ville est unique), le laboratoire n'est pas la société, et toute forme

\section{Notamment Bruno Latour}

9 E. Husserl, La crise des sciences européennes, 1936.

10 Chercheurs dans le domaine des OGM, nanotechnologies etc. qui nous promettent la vie éternelle, le contrôle total de la matière etc. Voir notamment le rapport J.-P. Dupuy \& F. Roure, Les nanotechnologies : éthique et prospective industrielle, 2004.

11 P. Roqueplo, Entre savoir et décision, l'expertise scientifique, Sciences en Question, INRA éditions, 1997

12 P. Roqueplo, Les experts sont formels, Paris, Autrement, 1992.

13 Voir notamment la discussion entre legein et teukhein chez C. Castoriadis, L'institution imaginaire de la société, Paris, Seuil, 1975.

14 B. Stiegler, La technique et le temps, Paris, Galilée, 1998.

15 Voir le coût croissant du transfert des supports physiques et matériels divers sous support numérique et l'inflation des budgets de « transcodage » et de «trans-archivage » 
d'investigation n'est pas autorisée - par exemple dans le cas de l'expérimentation humaine ou des problématiques de précaution. L'expérimentation OGM en plein champ pose des problèmes si les citoyens estiment qu'elle en pose. La problématique du risque est certes dépendante de connaissances mais surtout de valeurs. Si la société choisit de ne pas prendre ce risque-là, pour différentes raisons, c'est son droit le plus strict.

On le voit, quand on sort de la vision idyllique du chercheur " désintéressé », la garantie d'un financement public et d'une critique entre pairs ne suffit pas à garantir une indépendance qui soit autre chose qu'un corporatisme policé. Difficile de mettre ces réalités sur le dos de la privatisation ou du pouvoir excessif de l'administration centrale. La tendance dominante, au sein des mouvements sociaux et plus généralement des services publics, est malheureusement de nier le problème, comme en témoigne l'épisode Xavier Dunézat ${ }^{16}$. Les EC et leurs syndicats sont convaincus que poser cette question revient à mettre en doute l'un des piliers principaux de leur stratégie de résistance. L'indépendance par les statuts, le salaire et l'évaluation entre pairs suffit. C'est la garantie suffisante d'une liberté de penser. Pour eux, mettre en cause cette liberté, c'est remettre en cause la démocratie. C'est vouloir une science lyssenkiste ou une "téléthonisation » de la recherche, qui définirait ce qu'est le monde sur des critères politiques et donc idéologiques, ou une science " utilitariste ", engluée dans les applications pratiques, sans recul sur son action. Notre thèse au contraire est que ne pas aborder cette question fragilise les mouvements progressistes, car, en s'appuyant sur une espèce de méfiance diffuse envers le pouvoir arrogant de "la science " et "les profs », le gouvernement a beau jeu de jouer les corps intermédiaires contre la base, ce qui permet ensuite de trier pour ne retenir que ceux qui sont politiquement utiles. En ne défendant que les moyens, les statuts, le caractère public de la recherche et la performance évaluée "entre pairs ", l'université risque de ne pas réussir à donner envie aux citoyens de la soutenir; elle donne le bâton pour se faire battre avec la stratégie de Nicolas Sarkozy : jouer le peuple contre les corps intermédiaires, afin d'obtenir de ces derniers qu'ils se mettent dans l'ordre de bataille voulu. Si cette stratégie perdurait de la part des universitaires et de leurs organisations représentatives, ils ne pourront se plaindre, à court terme, d'être coupés de toutes les intelligences qui leur sont extérieures, qui portent sur eux un regard bienveillant, respectueux et chaleureux et qui surtout demeurent, en dernier recours, les seules forces de rappel vers des projets d'humanité où les seules contingences finalisées ne détermineront pas l'ensemble du corps doctrinal d'enseignement et de formation. Sans la prise à bras le corps de cette nécessité de dialogue permanent avec l'extérieur, qui aura des répercussions sur le contrat social sur lequel s'appuieront les projets à venir, l'Université sapera ni plus ni moins que toutes les bases qui fondent ses légitimités.

A partir de là, peut-on faire quelques propositions sur l'arrangement institutionnel qui serait le mieux susceptible de garantir un enseignement et une recherche de qualité, libérée de toute pression financière ou idéologique?

\section{Quel est le rôle de l'université ?}

La littérature et l'opinion commune attribuent à l'université quatre rôles possibles : sélectionner les cerveaux et reproduire l'idéologie dominante (Bourdieu), former des professionnels (vision utilitaire), développer l'universalisme et l'encyclopédisme, l'esprit critique, la recherche de la vérité pour elle-même (savoir "désintéressé " au sens de Humboldt), et la transmission de savoirs " scientifiques », réputés vrais et vérifiés, contre le savoir « profane ».

La thèse défendue ici est celle-là : ce qui est en jeu, pour garantir la liberté de penser - car c'est bien de cela dont il s'agit -, est l'existence d'un lieu vide de pouvoir, au sens que Claude Lefort donnait à cette fonction dans l'organisation d'un pouvoir démocratique ${ }^{17}$. L'enjeu est de préserver le savoir de toute mainmise par l'Etat, le Marché ou la Société Civile; la seule manière de le faire a

16 http://www.liens-socio.org//MG/pdf/dossiers liens socio 06 dunezat.pdf . Voir l'analyse faite par Numa Murard, La parenthèse (Xavier Dunézat) - pour l'émergēnce d'un débat (vraiment) contradictoire sur l'université, Mouvements, n55-56, pp46-52.

17 C. Lefort, L'invention démocratique, Paris, fayard, 1981. 

326.

été définie par Montesquieu : l'équilibre des pouvoirs. Les EC ne peuvent garantir aucune réelle indépendance ou liberté de penser s'ils n'en font qu'un enjeu corporatiste. L'université ne peut se poser en détentrice de la liberté de penser sans aussitôt en détruire l'existence, puisque s'approprier cette liberté c'est de facto en réduire la portée - et donc l'abolir tout court. Le cas des lanceurs d'alerte illustre parfaitement cette problématique. Des chercheurs comme Christian Vélot n'ont pas été inquiétés par un pouvoir privé mais par la direction d'un institut public; il n'a pas été soutenu par ses pairs mais par des organisations issues de la société civile, qu'on entendra ici au sens des organisations à but non lucratif. L'indépendance de l'enseignement et de la recherche ne peuvent tout simplement pas exister sans dialogue constant avec les différentes parties de la société, notamment celles qui sont aujourd'hui très largement exclues : association, PME, artisans etc. Seule l'épreuve de la discussion publique, pour reprendre l'argumentation d'Habermas, permet de faire un usage réellement critique de la raison ${ }^{18}$ - et non le repli sur des communautés spécialisées qui ont tendance au contraire à se bureaucratiser et à se reproduire, phénomène qui les rend hautement instrumentalisables, souvent à leur insu.

De ceci découle quelques conséquences.

La première est que la liberté de trouver des réponses n'a pas à être contrôlée - sauf évidemment par l'éthique, à nouveau : expérimentation humaine, protection de la vie personnelle etc. Le droit doit être le fait de toutes les parties de la société. La liberté de poser des questions, par contre, ne peut être réellement critique que si elle est collectivement organisée. Sans cela il se formera toujours de petites castes dont le souci est de rester à l'abri des critiques. Le scientifique dans son laboratoire ne jouit pas d'une autonomie spontanée, il reste pris dans ses préjugés, ses cercles familiaux, professionnels, son aveuglement disciplinaire etc. tant qu'il n'enracine pas son savoir dans le monde-de-la-vie social. II s'agit de protéger les EC contre leur possible souci de plaire aux pouvoirs en place et de mettre leur expertise au service de leur légitimation. L'autorité dont jouit le scientifique doit être confinée par des contre-pouvoirs. On suivra ici une partie des recommandations de Bruno Latour au sujet de la science en plein air et les forums hybrides. La preuve devrait être administrée d'une manière compréhensible pour le " profane » - car ce sont eux qui sont à convaincre. Concrètement les instances stratégiques telles que l'ANR, les conseils de programme etc. devraient, à chaque échelon de gouvernance (département, région etc.) faire de leur bilan annuel ou pluriannuel l'objet d'une conférence publique, et étayer leur stratégie à venir par des «conventions de citoyens" ainsi que le propose la Fondation Sciences Citoyennes ${ }^{19}$.

La seconde est que cette liberté ne serait rien sans une liberté de circulation de l'information, autre élément clé de la démocratie. L'exigence de véracité des informations n'est pas un privilège des scientifiques, elle est aussi demandée des journalistes etc. La circulation des savoirs doit être assurée par des instruments de vulgarisation de qualité, eux aussi non assujettis aux pouvoirs en place et donc en particulier de la logique de profit. La discussion ne doit pas rester confinée aux revues scientifiques. La liberté de penser implique de former des journalistes scientifiques et d'ancrer les enseignants dans la recherche, sous toutes ses formes. Des financements publics pérennes doivent être prévus pour les journaux qui choisissent de mettre en place un tel service public.

Troisième conséquence : organiser le lien de l'université avec certains secteurs de la société civile particulièrement critiques envers le pouvoir, qu'il s'agisse des artisans et PME ou des associations à but non lucratif. "La société » qui passe commande aux EC, aujourd'hui, se résume aux grandes entreprises et l'Etat d'où un manque de critique envers ces deux instances, un décalage croissant avec les aspirations des citoyens et un rejet de certaines innovations telles que les OGM, le nucléaire ou les nanotechnologies. L'articulation avec ces forces passe alors par une université plus " participative ${ }^{20}$, autrement dit ouvrir l'université, d'une part, et reconnaître la production de savoirs formalisés par d'autres producteurs que les détenteurs du statut de chercheur. En pratique,

18 J. Habermas, Théorie de l'agir communicationnel, Paris, Fayard, 1987.

19 http://sciencescitoyennes.org/spip.php?article1654, article publié par Jacques Testart, Michel Callon, Marie-Angèle Hermitte et Dominique Rousseau dans Libération le 26 novembre 2007.

20 A. Rubiao, Une université " participative »? Réflexions à partir du programme " Pôles de citoyenneté », Mouvements, $n^{\circ} 55-56$. 
cela signifie soutenir les 'outils' extra-universitaires tels que: Fondation Polanyi, Fondation Sciences citoyennes, Collège de Philosophie, CRIIRAD etc. et bien d'autres, et faciliter leur inclusion dans les programmes de recherche existants. Etablir un relevé des opportunités et des espaces existants pourrait constituer une tâche de première importance. Davantage de procédures de type conventions de citoyens, débats publics, y compris pour orienter la recherche et l'enseignement. Les projets PICRI, où les associations comme des coopératives peuvent être parties-prenantes de recherches formelles etc. Nous ne nous leurrons pas et savons pertinemment que les premiers acteurs de l'évolution du système universitaire qui peuvent aller dans ce sens ne seront pas les universitaires mais les collectivités territoriales et les élus.

\section{Des propositions concrètes}

Approfondissons un peu la question des pistes de sortie et des éléments de solutions. Certains sont de la responsabilité des acteurs de l'Université, d'autres de différents acteurs sociaux et culturels, d'autres enfin sont clairement dans les mains des leaders politiques. Avant de revenir sur ce dernier point, un mot pour qu'on ne méprenne pas le sens de ce qui pourrait apparaître comme une incartade ou une voie d'inconséquent.

II s'agit ici de prendre la mesure de deux schismes historiques que les mondes du savoir ont vécu et nourri : celui du "penser» et du «faire » pour reprendre les propos de Dominique Pestre (2009) qui féconda une dépolitisation lente mais inexorable de la corporation des scientifiques, chercheurs et ingénieurs ; celui du spirituel et du scientifique enfin, qui évacue la question du sens de la pensée, du sens de tous les métiers de la connaissance. Dans le premier cas, c'est la question du lien entre savoir et démocratie que nous devons affronter, mais recontextualisé, sans se goberger de grands mots mais de perspectives sociales, économiques et politiques très concrètes. Dans le second cas, la crise actuelle illustre le saut que nos sociétés sont en train de franchir : celui du besoin " congénital » d'un méta-discours. Qu'il fût théologique à ses origines, philosophiques par la suite, scientiste plus récemment, l'univers des savoirs ne peut faire l'économie d'un " encastrement » spirituel et laïque - sous peine de nourrir une crise du sens aux effets incommensurables. Nous faisons le pari que nous assistons aujourd'hui à l'apparition d'un paradigme " écologique " au sens très large et qui dépasse les disciplines éponymes. Ecologie des savoirs (profanes, techniques et scientifiques compris), biodiversité des disciplines, coopération conceptuelle etc.... Les révolutions les plus douces - ce souterrain des choses aurait dit Walter Benjamin - sont presque toujours en définitive les plus puissantes ${ }^{21}$. Elles sont en marche en permanence et affleurent à la surface quand elles ne peuvent plus masquer ni leur puissance ni leur force ni leur maturité ni leur " évidence ». Lorsque les temps et les mœurs sont mûrs. De cette évolution du paysage mental des sociétés naissent de nouvelles normes et institutions. Sur ce sujet, le président étasunien Barak Obama est le seul à ce jour à avoir résolument établi le lien, en termes de politiques publiques, entre crises, enjeux écologiques, besoins sociaux et éducation. II est, en l'espèce, un exemple à suivre. II réencastre l'institution universitaire dans la société qui l'accueille. Il lui donne des claires perspectives, motive ses acteurs, engage et sollicite des débats dans les institutions. Ni l'Université ni le système éducatif n'y sont perçus comme une charge sur les finances publiques mais comme un des éléments déterminants des solutions à venir dans des sociétés qui abordent, fiévreuses, une ère nouvelle. N'ayant pas d'informations précises sur l'investissement colossal que le gouvernement chinois annonce (près de 1000 milliards d'euros), il est difficile d'y porter un regard précis. Toutefois il est fort probable que le modèle de développement devrait être remis en question. Dans un registre différent, mais tout aussi porteur d'évolutions profondes sur le long terme : la décision du président bolivien Evo Moralès, l'an passé, de créer la première université des savoirs indigènes. Ces deux initiatives sont à prendre comme les deux faces, économique et culturelle, d'un même enjeu : la place des savoirs, de tous les savoirs et savoir-faire, dans des sociétés en complète évolution.

Au-delà des nécessaires réformes de l'appareil universitaire lui-même, il apparaît de plus en plus

21 Qu'on songe par analogie à la plus grande révolution française du XXème siècle qui ne fit aucun mort, une révolution pourtant hautement sensible puisqu'elle touche à la terre, au foncier : le remembrement 
évident, comme cela le fut à d'autres moments de l'histoire de cette institution qu'une grande partie des propositions viendra de l'extérieur, vient déjà de l'extérieur. II existe aujourd'hui une foule d'espaces sociaux où s'échangent et se transmettent mais aussi - et c'est assez nouveau - se produisent des connaissances de qualité. Le don gratuit de savoirs profanes et scientifiques n'a jamais été aussi massif qu'aujourd'hui : universités populaires, Wiki, participation de réseaux " profanes » à des recherches, réseaux d'échanges des savoirs, cafés scientifiques, débats locaux, etc. Jamais les savoirs n'ont été autant diffusés et aussi bien, dans l'ensemble. L'Université ne peut se désintéresser de ces évolutions d'autant que dans les faits nombre d'universitaires et d'étudiants sont embarqués dans ces dynamiques. Etre demeuré à l'écart de l'évolution de ces "demandes sociales" pourrait expliquer en partie le fait que la légitimité de l'institution universitaire se soit progressivement réduite à la dispense de diplômes qui, par rétroaction, nourrit une relation aux savoirs biaisée et réduite à sa dimension la moins savante et la plus utilitariste qui puisse être : le maintien d'une autorité de principe, fondée sur le diplôme. La demande de savoirs non utilitaires n'a jamais été aussi massive, et l'institution semble éloignée de ce phénomène.

Quelle alternative avancent les enseignants-chercheurs dans le débat sur le maintien d'une « ligne de défense " vitale des recherches non finalisées, sur la justification d'un enseignement des " humanités" ? La formation à une citoyenneté moderne. Cette "idée en dernier recours " est séduisante, mais, pour paraphraser Cyrano de Bergerac : "c'est un peu court ». En effet comment concilier dépolitisation des professionnels des savoirs et ambition d'un projet de citoyenneté ? L'équation reste sans solution et les conditions culturelles de l'échec sont réunies. II faut toujours manier les idées plus grandes que soi avec parcimonie et modestie. A cette heure, l'Université ne semble pas armée pour donner des leçons, à tous les sens du terme, sur ce que doit et peut être cette citoyenne moderne. Pour autant, nous avons là, entre les mains, probablement la seule hypothèse sérieuse du socle de projet politique acceptable et pertinent pour une université au XXI ${ }^{\text {ème }}$ siècle.

Prenons un exemple qui devrait trouver un écho politique dans la communauté des EC : l'évolution du statut de la connaissance. Face aux offensives tous azimuts de brevetabilité le plus en aval possible, la dernière véritable révolution dans le statut juridique de la connaissance fut le fait de militants du libre, qui à force de hasards et d'expérimentations ont favorisé l'apparition d'un nouveau régime de brevet: les licences libres. Elles sont désormais légion. Certains instituts français ont même développé le leur (CeCILL pour "CEA CNRS INRIA Logiciel Libre »). On ne perçoit pas de mouvement au sein de l'université qui discuterait résolument son implication dans cette lutte cruciale pour leurs métiers que constitue la "bataille des biens communs". Peu d'attention stratégique à l'endroit des promoteurs des Open review, des Open course. Bref, tout ce qui permet d'abaisser le ticket d'entrée et d'accès au savoir et à la connaissance au lieu d'en faire un enjeu de rareté, et donc de guerre. C'est en ce sens, qu'on doit analyser, aussi, à la fois la faible politisation du milieu de la recherche, comme de sa schizophrénie à revendiquer un rôle majeur dans une " construction de la citoyenneté " sans se préoccuper, a minima, des tendances inquiétantes, mais porteuses de débats profonds sur ce qui constitue, en somme, leur cœur de métier: le statut du savoir et la promotion d'un savoir ouvert, décomplexé, accessible, véritablement « libéré ».

Les ambitions "citoyennes" des universités auront leur cohérence si et seulement si les établissements autonomes et décentralisés qui perdureront inscrivent au cœur de leurs préoccupations, au même titre qu'une adaptation à la construction d'une économie responsable et soutenable, les enjeux démocratiques; si et seulement si cette ambition irrigue toutes les politiques et orientations internes de là à insuffler un profond état d'esprit démocratique, une culture de l'esprit critique, un appétit pour la dispute, un souci de la contradiction et de l'argumentation raisonnée, une ouverture interculturelle au "penser large " et à la " pensée du large »; si et seulement si on situe la confiance dans les jeunes générations comme un principe a priori, si on sollicite et promeut les innovations sociales, politiques et culturelles à l'intérieur même de l'Université, champ pris dans son acception de terreau, de friche, d'espace d'expérimentation. En l'espèce, un grand nombre de disciplines pourraient concourir à enrichir l'enseignement et la 
pratique. Cette ambition devrait se décliner dans la gouvernance démocratique de l'institution, dans les différentes strates décisionnaires, dans le décloisonnement des corpus, dans la promotion de recherches dites hybrides/participatives et dans la nécessité d'expérimentations pédagogiques.

Fort heureusement, quelques signaux sont émis dans le mouvement de 2009. Pour ne prendre que quelques exemples, ceux de l'Université de Nanterre-Paris $X$ ou de Paris VIII. Plusieurs professeurs qui suivent le mouvement au plus près sont étonnés de l'ouverture d'esprit des étudiants et enseignants-chercheurs, de ce besoin de " mettre tout sur la table ", d'aborder sans trop d'idées préconçues l'ensemble des enjeux. Si nous pouvions a minima vérifier que nous assistons aux prémices d'une généralisation de la problématique, alors il serait de notre responsabilité de prendre une initiative en ce domaine. De fédérer des acteurs, de leur permettre d'instituer un lieu d'échange et de débats permanents sur ces enjeux véritablement historiques.

II est somme toute bien naturel et en définitive systématique que les soubresauts de l'Histoire rattrapent les institutions d'enseignement supérieur. Souvent dans la douleur pour ce qui est du $X X^{\text {ème }}$ siècle. Qu'on se rappelle les années 30 de triste mémoire qui posèrent en Allemagne et en France des questions essentielles - et différenciées. Dans le premier cas, allemand, la faillite intellectuelle des élites et des valeurs véhiculées par une société fut totale. Une société composée d'individus largement éduquée pouvait agir de façon totalement inhumaine. Les savoirs scientifiques, qu'ils soient ancrés dans les sciences de la nature ou dans les sciences humaines et sociales ne prédisposent pas - si tant qu'ils y prédisposassent jamais - à l'humanisation de nos civilisations. Toutes les avancées éthiques et morales acquises depuis lors sont à ranger au chapitre des effets de ces crimes historiques de la pensée à l'aune desquels nous mesurons désormais le niveau de notre incertitude et de nos doutes: principe de précaution, moratoire, résistance à la dictature techniciste et scientiste...

En France, dans un tout autre ordre d'idée, toujours en 1945, le jugement d'un Marc Bloch fut sans appel: "qu'il s'agisse de stratégie, de pratique administrative ou, simplement, de résistance morale, notre effondrement a été avant tout, chez nos dirigeants et (pourquoi ne pas avoir le courage de l'avouer?) dans une partie de notre peuple, une défaite à la fois de l'intelligence et du caractère. C'est dire que, parmi ses causes profondes, les insuffisances de la formation que notre société donnait à ses jeunes ont figuré au premier rang. (...) II importe que pour l'éducation de ses jeunes comme pour le développement permanent de la culture dans l'ensemble de ses citoyens, la France de demain sache dépenser incomparablement plus qu'elle ne s'y est résigné jusqu'ici. $»^{22}$

Aujourd'hui, nous assistons à une nouvelle faillite de la formation de nos élites. Tous les appareils de sélections nationaux ont produit des hommes et des femmes hautement éduqués, en provenance quasi-exclusive des institutions universitaires les plus renommées au monde: étatsuniennes, britanniques, japonaises, indiennes comme de bien d'autres pays et, bien entendu, nos grandes écoles et nos grands corps de l'Etat. Progressivement déracinées de leurs responsabilités au service du bien public et du bien commun, elles s'installèrent au cœur des grandes institutions financières, au cœur des valeurs de leur temps, au cœur de leurs intérêts de classe. Ils en furent les principaux agents innovateurs. On pense ici avant tout au rôle fondamental des mathématiciens financiers qui sont en grande partie responsables des cataclysmes actuels. Pour reprendre les propos de Pierre-Henri Gouyon, on ne peut pas ne pas traiter la question centrale du rapport entre les lieux de la production et d'enseignement de savoirs et le capitalisme.

Comme n'importe quel autre pays, la France possède des atouts et des faiblesses propres à son histoire. Déjà, en 1793, les révolutionnaires, constatant l'état de déréliction de l'Université, l'avait purement et simplement abolie. Ne l'oublions pas, une société française sans université fut possible dans les temps "modernes ». On peut sans grossir le trait affirmer que l'Université en France n'a jamais véritablement su ni pu intégrer la plupart des évolutions de son temps. La séparation d'avec le technique par exemple se paie dans la durée. L'apparition progressive dans le paysage de l'enseignement supérieur des Grandes Ecoles, du Collège de France, de l'Ecole

22 Cité dans M.-L. \& J.-M. De Montrémy, L’Europe des universités, Paris, Gallimard, 2008. 
Pratique des Hautes Etudes (1868), de l'Ecole libre des sciences politiques (Sciences Po, 1871), du CNRS en 1939, de l'ENA en 1945, des écoles d'ingénieurs puis des IUT s'explique soit par la faillite d'un système d'enseignement supérieur soit par une incapacité à articuler aux curriculum en place de nouvelles nécessités, de nouveaux savoirs et savoir-faire constitués, de nouveaux besoins ainsi que de nouvelles méthodologies pédagogiques. II ne s'agit pas d'un procès mais d'un constat: I'Université et ses acteurs sont en grande partie responsables, devant l'Histoire, du morcèlement de l'enseignement supérieur français.

A ce morcellement s'ajoute la seconde métastase de l'Université "à la française ». Elle demeure toujours engluée dans la période ouverte à la fin des années 1960 : celle de la massification. Nous sommes toujours en présence d'un tiraillement du milieu, entre une posture à la Raymond Aron (démissionnaire de la Sorbonne en 1967, dépité devant les affres d'une démocratisation impliquant selon lui, mécaniquement, une qualité et une exigence dépréciées) et un souci de franchir " le palier éducatif de haut niveau " pour reprendre la récente formule d'Emmanuel Todd ${ }^{23}$. En quarante ans, aucune politique publique ni mouvement d'ensemble n'ont permis de résoudre cette apparente contradiction de l'articulation entre excellence et massification. Cette ambition élitaire comme aurait dit Jean Vilard est-elle inaccessible?

La tradition universitaire veut que l'on privilégie le travail personnel, non dirigé, autonome : stimuler l'esprit critique, donner le goût d'apprendre, d'apprendre à apprendre, sensibiliser à la culture de la curiosité et de l'ouverture. En somme, responsabiliser les jeunes face à leur propre formation. Mais quand on dit responsabiliser, ceci signifierait qu'il y eût un temps où la corporation des enseignants aurait effectué un travail pro-actif en ce sens. Rien n'est moins vrai. Au temps de l'Université d'élite le projet universitaire n'était pas questionné, il allait de soi. On savait ce qu'on fichait là. L'homogénéité du milieu, la cooptation culturelle agissaient comme un ciment tacite. La période de la reproduction des élites ne posait pas grande question. Désormais l'accompagnement, le dialogue entre enseignants et étudiants est requis, résolument, car les " publics » ne sont plus les mêmes et ne le seront plus à courts et moyens termes. Les enseignants du supérieur, tout du moins ceux de l'Université, sont peu formés à ces enjeux. Ils ont exclu de leurs réflexions les enjeux d'ingénierie pédagogique, autre gros mot dans le paysage français, alors qu'il va de luimême dans tous les autres pays développés, des Etats-Unis au Japon. Bien entendu qu'une nouvelle ingénierie pédagogique est requise en phase de massification; bien entendu qu'il n'est pas aberrant de penser des processus d' " industrialisation de qualité " de la transmission des savoirs. II n'y a que celles et ceux qui sont rétifs aux contraintes économiques qui ne veulent pas intégrer cette dimension dans les débats. Mais ils sont probablement bien plus rares qu'on ne l'affirme. Des pistes nombreuses existent qui sont déjà largement défrichées dans une multitude de sites français et étrangers. Certaines expérimentations à petite échelle sont plus médiatisées. On parle du succès de l'initiative de Sciences Po à l'adresse de jeunes issus des ZEP. Sans dénier nullement ce véritable succès et cette belle (et opportuniste) initiative, la question de son " industrialisation" se pose et, partant, la question des innovations requises en ingénierie pédagogique.

On peut évoquer ici par analogie la loi Pisani de 1965 sur l'enseignement agricole. Au-delà des postulats idéologiques de son temps (nourrir la France, productivisme effréné), elle fut une véritable révolution dans l'enseignement agricole secondaire et supérieur. Le directeur de l'établissement peut être comparé à ce que pourra être le président des établissements universitaires. Grâce à l'accueil d'un enseignement socioculturel, des dialogues naturels s'opèrent entre les filières et le territoire. Ce qui ne voulut pas dire une «fermeture " sur le local ni une féodalisation du projet éducatif. Le juste équilibre entre la normativité d'un enseignement national et l'adaptation à une réalité locale semble avoir été bénéfique. Si l'on ajoute à ce succinct bilan l'obligation faite d'un projet à caractère international, et sans évacuer nombre d'errements propres à tout système, personne, pas plus les enseignants, que les élèves, que les parents, que les acteurs de la filière, ne se plaint aujourd'hui de ce " modèle ». Bien au contraire. On reste stupéfait et triste de l'écart stratosphérique d'avec les établissements généralistes (entendez ici les lycées).

23 E. Todd, Après la démocratie, Paris, Gallimard, 2008 

326.

Nous faisons l'hypothèse que cette libération potentielle des énergies des universités, contenues tacitement dans les réformes en cours, sont de nature à donner du souffle à des établissements dont plus personne ne conteste le fait qu'un contrôle exclusivement national mène à leur perte.

II est faux de considérer l'érosion du nombre d'inscrits dans les sciences naturelles ou dans d'autres disciplines telles que le droit ou les lettres comme le symptôme d'un désintérêt. II s'agit avant tout d'une désaffection, de choix rationnels effectués par les lycéens et leurs familles. La rentabilité d'études longues est posée. Si l'on rajoute à cela un second paramètre déterminant qui est la dépréciation de soi, la conviction " de ne pas être au niveau » et " qu'on n'y arrivera pas " ${ }^{24}$ nous sommes face à un travail à réaliser, par des hommes et des femmes dont ce sera la mission nationale (et européenne), sur la motivation et la mobilisation des jeunes générations.

Dès lors, à notre sens, une alliance contractuelle avec l'éducation non formelle est requise. Ce sont les seuls acteurs facilement mobilisables, compétents, dont les rôles historiques furent de premier pan dans l'histoire éducative de notre pays. Le monde associatif et au premier chef celui de l'éducation populaire doit y trouver un ressourcement, un nouveau rôle historique. Pourquoi eux ? Car si les politiques publiques - en France notamment - continuent de jouer la carte du monopole du contrat social, si l'on continue à amalgamer service public et statut d'agent du service public, nous échouerons. Décentrer les enjeux des institutions préserve ces dernières tout en responsabilisant les acteurs sociaux. On ne peut, d'un côté, défendre un système éducatif républicain et laïque en demandant à la collectivité de ne pas faire reposer sur ses seules épaules l'ensemble des maux et des contradictions d'une société si c'est pour, dans le même temps, revendiquer le monopole et la centralisation des moyens, ainsi que le monopole du bon savoir. II faut en la matière plus qu'ailleurs de la cohérence. Résoudre les tensions qui pèsent sur l'Université suppose voire impose un élargissement de la base des co-responsables de son avenir. Les méthodes de l'éducation formelle constituent une plus-value indéniable. Elles y sont souples et dynamiques, l'ancrage social et démocratique des acteurs indéniable, précieux et surtout unique. Le tissu associatif sera probablement le premier partenaire naturel des universités pour peu que des régulations et des règles soient instituées. Nous pourrions tout à fait proposer, sur le modèle des contrats éducatifs locaux, un outil d'administration et de politique publique pertinent qui prendrait la forme d'un "contrat local d'éducation supérieure » (CLES), sous l'égide des collectivités territoriales, des institutions concernées et des acteurs sociaux et culturels. Le contenu de ces CLES devrait placer, en tête de chapitre, selon nous, la mobilisation d'énergies et de forces sociales à même de lutter contre l'augmentation de la démotivation et de la démobilisation des jeunes générations.

Pour la première fois depuis longtemps, l'avenir que notre société prépare à ses enfants est plus préoccupant et moins lumineux que celui de leurs parents. Dans son dernier ouvrage, Camille Peugny résume l'enjeu colossal que nous affrontons: "Dans la France des années 2000, connaître une moins bonne réussite sociale que ses parents n'est plus exceptionnel : c'est ne réalité statistique indiscutable mais une réalité sociale méconnue. Les générations nées au tournant des années 1960, confrontées aux effets prolongés de la crise économique, font face à une dégradation de leurs perspectives de mobilité sociale. Dans le même temps, leur niveau d'éducation continue d'augmenter. De ce décalage entre la formation et la mobilité sociale naît un intense sentiment de frustration qui a des conséquences sur l'expérience vécue par des " déclassés ", qui oscillent alors entre deux tentations : la rébellion et le retrait. " ${ }^{25}$

Le premier pôle d'une collaboration nouvelle et intelligente entre acteurs sociaux, éducatifs et institutions pourrait être à dix ans, de travailler spécifiquement sur cette dimension psycho-sociale : mobiliser et motiver les jeunes générations sur leurs propres potentialités et sur leur rôle dans la société. Ces enjeux d'« alphabétisation culturelle " convergent avec les nécessités de cohésion sociale. Cette question est bien entendue nouvelle pour l'enseignement supérieur, à quelques rares exceptions près (tel Paris VIII par exemple).

Les questions pédagogiques et sociales sont intrinsèquement liées. Evacuer la question sociale de

24 B. Convert, Les impasses de la démocratisation scolaire, Paris, Ed. Raisons d'agir, 2006

25 C. Peugny, Le déclassement, Paris, Grasset, 2009 

326.

l'avenir de l'enseignement supérieur est tout bonnement aberrant. Que l'université française n'ait réussi en plus d'un siècle et demi qu'à adosser aux cours magistraux des TD et des TP interroge et afflige en même temps.

Enfin, pour finir sur ces perspectives et les enjeux que nous devrions affronter, nous souhaitons aborder un thème à la frontière de l'épistémologie et de la pédagogie. Une des tendances les plus lourdes de la vie des laboratoires et d'équipes de recherche est à l'agrégation des forces intellectuelles interdisciplinaires. N'en déplaise à celles et ceux qui en parlent comme d'un marronnier. La part la plus éclairée de la communauté des scientifiques sait que nous affrontons cette question : une douce remise en question du primat réductionnisme et « disciplinariste ». Ceci a bien entendu des effets mécaniques sur les modes d'enseignement. Des textes émanant de hautes autorités scientifiques et éducatives japonaises et étasuniennes constatent le besoin de préparer des intelligences à cette convergence intellectuelle de disciplines à ce jour séparées (prenant appui sur des demandes des promoteurs des nanotechnologies, certes, mais ceci ne doit pas nous détourner de l'intérêt de ce thème). On peut faire la même analyse des impacts que le paradigme écologique (décliné en "éducation au développement durable ") sur les systèmes d'enseignements dès le primaire. Le réductionnisme, seul, est un frein à la préparation cognitive des jeunes générations. Ceci n'est pas une mince affaire. II est même possible, amusons-nous un instant, que les nanolâtres et les écolophiles puissent trouver, ici, un terrain d'entente : dépasser l'enseignement par discipline du premier âge jusqu'à l'Université. Refaire advenir, d'une façon réactualisée ce besoin de scientifiques multidisciplinaires de haut niveau, cet « esprit universel » si l'on veut ici reprendre le qualificatif utilisé lors de la mort de Pierre-Gilles de Gênes. Quel bel enjeu, non?

Ceci peut constituer sans aucun doute possible un horizon "programmatique " ambitieux et fédérateur. D'autant qu'il percute un autre sujet d'inquiétude : l'érosion du niveau de culture scientifique des professionnels du savoir eux-mêmes ${ }^{26}$. II n'y a plus de différence entre des scientifiques et des citoyens dits lambda. Les SHSistes sont assez ignorants de la technique ou des sciences de la nature comme les " hard scientists » sont plus que déculturés vis-à-vis de ces sciences si "molles». La place prééminente de la modélisation, l'explosion de l'utilisation des outils informatiques transforment le métier même de chercheur dans nombre de laboratoires, y compris dans des disciplines des SHS. Une part de plus en plus importante des chercheurs opère désormais plutôt en tant que super-ingénieur - rien de péjoratif ici. Une proportion immense des thésards et post-doctorants contractuels relève de cette catégorie-là. Leur relation intime et cognitive à la construction de leur propre savoir en est profondément modifiée. Nous n'avons aucun recul, par force, sur cette "culture du simulateur ». Un des moyens de contention de dérives déjà observées en maints endroits réside dans l'élaboration d'un régime d'enseignement et d'éducation à une "culture scientifique et humaniste de haut niveau ". Là encore des initiatives semblent débuter dans certaines universités ou autres lieux de formation (Paris VI, CNAM). Nul doute qu'elles feront école car elles permettent, précisément de dépasser les débats stériles qui oppose vocation de l'Université à formation tout au long de la vie, des salariés et citoyens en général. Mais ceci, sans nul doute, fera l'affaire d'autres articles.

Pour finir, au regard de la diversité des chantiers, nous ne pouvons que reprendre à notre compte la volonté d'enclencher enfin des réformes, ambitieuses pour notre communauté politique (national et européenne) qui méritent enfin leur nom, qui cessent sous les oripeaux des contre-réformes de prendre les vessies de certains irresponsables pour nos lanternes. Seule une dynamique d'Etats généraux, ouverts à tous les acteurs impliqués en soucieux de l'avenir de l'enseignement supérieur, est à même de donner satisfaction quant à la méthode et aux résultats attendus. Qui prendra l'initiative?

26 Jean-Marc Lévy-Leblond, Le partage de l'ignorance, in La vitesse de l'ombre, Seuil, 2006 